\title{
Age estimation using carpals: Study of a Slovenian sample to test Cameriere's method
}

\author{
Roberto Cameriere $^{\mathrm{a}, *}$, Luigi Ferrante ${ }^{\mathrm{b}}$, Branko Ermenc $^{\mathrm{c}}$, Dora Mirtella ${ }^{\mathrm{b}}$, Katja Štrus ${ }^{\mathrm{d}}$ \\ ${ }^{a}$ Institute of Forensic Medicine, University of Macerata, Via Don Minzoni 9, 62100 Macerata, Italy \\ ${ }^{\mathrm{b}}$ Institute of Microbiology and Biomedical Sciences, Polytechnic University of Marche, Ancona, Via Ranieri 65, 60100 Ancona, Italy \\ ${ }^{\mathrm{c}}$ Institute of Forensic Medicine, Faculty of Medicine, University of Ljubljana, Korytkova 2, 1000 Ljubljana, Slovenia \\ ${ }^{\mathrm{d}}$ Surgical Clinic Medical Centre, Zaloska 2, 1000 Ljubljana, Slovenia
}

Received 8 November 2006; received in revised form 2 February 2007; accepted 11 April 2007

Available online 23 May 2007

\begin{abstract}
Carpals are often used as age indicators. In a recent study, Cameriere et al. studied the use of the ratio between the total area of carpal bones and epiphyses of the ulna and radius (Bo) and carpals (Ca) as age indicators. The present study, of a sample of 158 Slovenian children and adolescents aged between 6 and 16 years, focused on analysing the best regression for age estimation. The regression model yielded the following equation: age $=-3.411+0.942 g+20.927(\mathrm{Bo} / \mathrm{Ca})$, and explained $91.6 \%$ of total variance $\left(R^{2}=0.916\right)$. The median of the absolute values of residuals (observed age minus predicted age) was 0.09 years, with a quartile deviation of 0.786 years, and a standard error of estimate of 0.658 years.

Comparisons between the previous equation referring to Slovenian children and the equivalent linear equation proposed by Cameriere et al. did not reveal any significant differences between the intercepts and slopes of the two linear models. These results suggested a common regression model for both Italian and Slovenian samples.

The common regression model, describing age as a linear function of gender and Bo/Ca ratio, yielded the following linear regression formula: age $=-2.907+0.408 g+20.757(\mathrm{Bo} / \mathrm{Ca})$. This model explained $86 \%$ of total variance $\left(R^{2}=0.86\right)$. The median of the absolute values of residuals (observed age minus predicted age) was 0.02 years, with a quartile deviation of 1.02 years and a standard error of estimate of 0.96 years.

(C) 2007 Elsevier Ireland Ltd. All rights reserved.
\end{abstract}

Keywords: Age determination; Carpals; Linear regression; Illegal immigration

\section{Introduction}

Adolescence is a period of life marked by several changes of the body, and a number of these changes may be used as age indicators. In particular anthropometric data, signs of sexual maturation and mineralisation of bones, are used [1,2].

As regards bone mineralisation, the growth of several parts of the body and also the appearance of ossification centres, their morphology, size, and fusion can also be used for age estimation. Clavicle, knee, hand, and teeth are the most frequently studied. Knee and clavicle [3-6] are used only for their fusion of ossification and specifically to evacuate ages of 18 or 21 years, respectively. Conversely teeth and hands [7-12] may be studied throughout their growth and can be used as age

\footnotetext{
* Corresponding author. Tel.: +39 0733 2582625; fax: +390733 2582635 .

E-mail address: r.cameriere@unimc.it (R. Cameriere).
}

indicators in adolescence. In orthodontic treatment, biological evaluation or forensic cases, hands and teeth are the parts most frequently used as age indicators.

The judicial system often demands that a child of unknown age be assigned an age to ensure that appropriate procedures are observed in the processing of a court case. In European countries, the age threshold with regard to criminal responsibility ranges from a minimum of 7 years of age in Switzerland and Ireland to 21 in Germany.

Hands, and especially carpals, have been used as age indicators in several studies. Examination is facilitated by the absence of other hard tissues and the large number of bones, making this area preferential for age evaluation.

Cameriere et al. [13] applied the ratio between the total area of carpal bones and epiphyses of the ulna and radius (Bo) and carpals $(\mathrm{Ca})$ as age indicators. The regression model, describing age as a linear function of the ratio $\mathrm{Bo} / \mathrm{Ca}$, yielded the following equation: age $=-3.253+0.719 g+20.610(\mathrm{Bo} / \mathrm{Ca})$, 
Table 1

Age and gender distribution of Slovenian sample

\begin{tabular}{lccr}
\hline Age (years) & No. of females & No. of males & Total \\
\hline $6-7$ & 3 & 2 & 5 \\
$8-9$ & 6 & 11 & 17 \\
$10-11$ & 17 & 9 & 26 \\
$12-13$ & 32 & 34 & 66 \\
$14-16$ & 17 & 27 & 44 \\
Total & 75 & 83 & 158 \\
\hline
\end{tabular}

and explained $83 \%$ of the total variance $\left(R^{2}=0.83\right)$. The median of the absolute values of residuals (observed age minus predicted age) was 0.08 years, with a quartile deviation of 1.59 years, and a standard error of estimate of 1.19 years.

The aim of this study was to test this method on a Slovenian sample, and to provide a specific formula.

The equation was subsequently compared with the linear equation [13] to establish whether a common regression model for both Italian and Slovenian samples could be developed.

\section{Materials and methods}

The design of this study was a retrospective cross-sectional study of radiographs. X-rays of wrist and hand taken from 159 Caucasian Slovenian children ( 83 males, 76 females) aged between 6 and 16 years were analysed (Table 1). All subjects were orthodontic patients and orthopantograms (OPGs) were made from 2001 until 2004. X-rays in the postero-anterior projection were taken of the left hand, with fingers slightly splayed. X-rays were produced in standardised conditions following the indications of TW3. No subject included in the study displayed any growth disorders. X-ray images were processed by a computer-aided drafting (CAD) program (Adobe Photoshop 7, Adobe System Inc., 2001).

The method followed the study previously published by Cameriere et al. in 2005. Briefly, the carpal area (Ca) and epiphyses of ulna and radius were

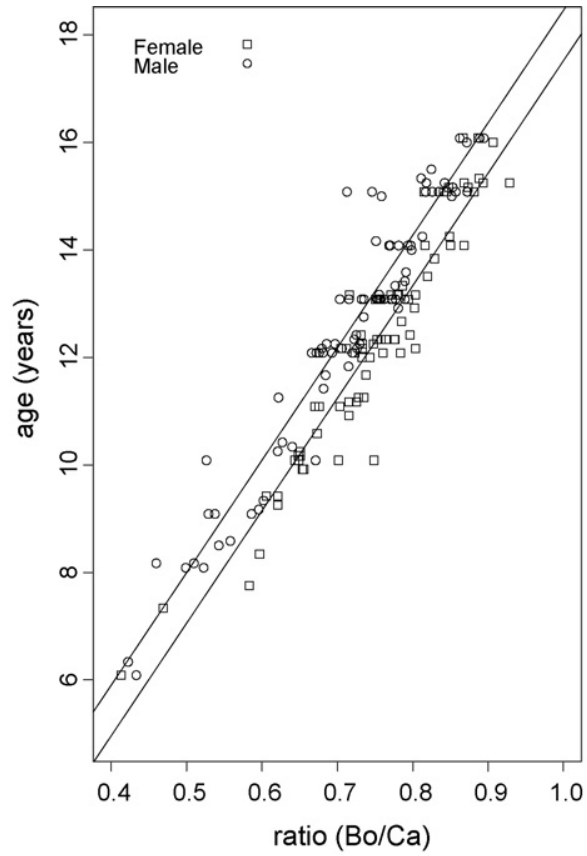

Table 2

ANCOVA table for model

\begin{tabular}{lrrrrr}
\hline & d.f. & \multicolumn{1}{c}{ SS } & \multicolumn{1}{c}{ MS } & $F$-Value & \multicolumn{1}{c}{$p$} \\
\hline$G$ & 1 & 2.938 & 2.938 & 6.75 & 0.010 \\
$\mathrm{Bo} / \mathrm{Ca}$ & 1 & 726.643 & 726.643 & 1670.19 & $<0.001$ \\
$g:$ Bo/Ca & 1 & 0.071 & 0.071 & 0.16 & 0.687 \\
Residuals & 154 & 67.00 & 0.435 & & \\
\hline
\end{tabular}

identified and defined by the polygonal lasso instrument of Adobe Photoshop 7 software. The pixels of the area were computed by CAD and are presented as a histogram.

The areas of the eight carpal bones were selected, and the pixel areas were calculated and added together to yield the global values of bone areas (Bo). When two bones overlapped, the common area was calculated only once. The $\mathrm{Bo} / \mathrm{Ca}$ ratio between total area of bones and carpal area was calculated and used for linear regression analysis with chronological age as dependent variable, and $\mathrm{Bo} / \mathrm{Ca}$ and gender as predictors. Subsequently, the Slovenian sample was pooled with the Italian sample evaluated in Ref. [13], made up of 150 Italian children and adolescents ( 89 males, 61 females) aged between 5 and 17 years, and a regression analysis was performed to this pooled sample.

\section{Results}

Using the data from the Slovenian sample, the analysis of covariance (Table 2) shows a significant difference in the intercept of the linear model between males and females whereas the slope in the regression models for males and females is not significant ( $p=0.687$ ), which corresponds to the parallel regression lines.

The parameter estimates of the model with equal slope but separate intercepts for male and female individuals are listed in Table 3.

The regression model, describing age as a linear function of gender and the $\mathrm{Bo} / \mathrm{Ca}$ ratio for the Slovenian sample, yielded

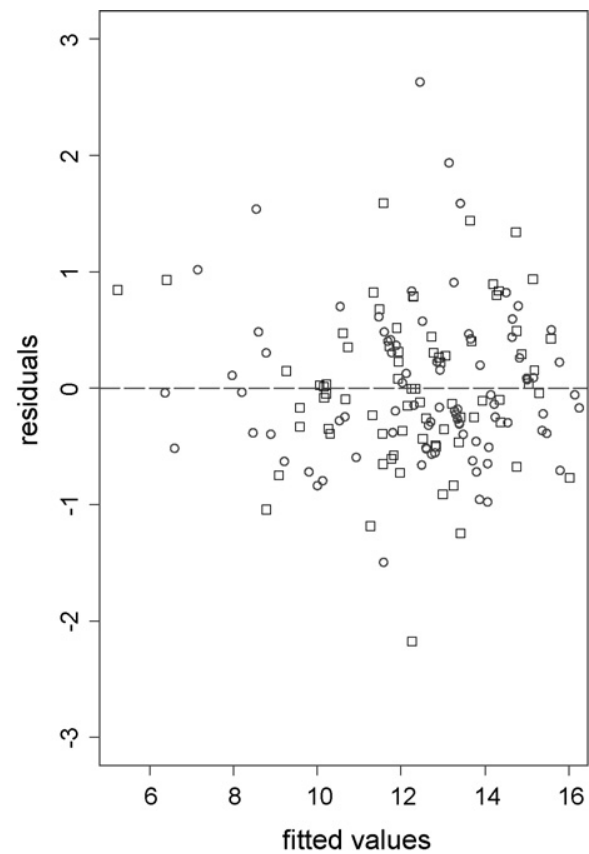

Fig. 1. Plots of data and regression lines (left panel) and residuals against fitted values (right panel) using a simple linear regression model (Eq. (1)). 
Table 3

Regression analysis predicting chronological age from gender and $\mathrm{Bo} / \mathrm{Ca}$ ratio

\begin{tabular}{lllrl}
\hline Coefficients & \multicolumn{1}{c}{ Value } & S.E. & $t$-Value & $p$ \\
\hline Intercept & -3.411 & 0.390 & -8.743 & $<0.001$ \\
$g$ & 0.942 & 0.106 & 8.886 & $<0.001$ \\
Slope & 20.927 & 0.511 & 40.979 & $<0.001$ \\
\hline
\end{tabular}

the following linear regression formula (Fig. 1, left panel):

age $=-3.411+0.942 g+20.927\left(\frac{\mathrm{Bo}}{\mathrm{Ca}}\right)$

This model explained $91.6 \%$ of total variance $\left(R^{2}=0.916\right)$. The median of the absolute values of residuals (observed age minus predicted age) was 0.09 years, with a quartile deviation of 0.786 years and a standard error of estimate of 0.658 years.

The residual plot (Fig. 1, right panel) shows no obvious pattern, and data points did not plot outside the expected boundaries. The regression lines (Fig. 1, left panel) show that the regression model fits the trend of the data reasonably well. Hence, both diagnostic plots support the chosen model.

Comparison between Eq. (1) and the equivalent linear equation in Ref. [13] showed non-significant differences between intercepts and slopes. These results suggested that we look for a single regression model for both Italian and Slovenian samples. Regression analysis confirmed that nationality did not affect the considered model $(p=0.074)$ and, accordingly, this covariate was dropped from the model. The estimated parameters are listed in Table 4.

The regression model for Italian and Slovenian individuals together, describing age as a linear function of gender and $\mathrm{Bo} /$ $\mathrm{Ca}$ ratio, yielded the following linear regression equation
Table 4

Regression analysis predicting chronological age from gender and $\mathrm{Bo} / \mathrm{Ca}$ ratio, for both Italian and Slovenian samples

\begin{tabular}{lrlrl}
\hline Coefficients & \multicolumn{1}{l}{ Value } & S.E. & $t$-Value & \multicolumn{1}{l}{$p$} \\
\hline Intercept & -2.907 & 0.350 & -8.308 & $<0.001$ \\
$g$ & 0.408 & 0.055 & 7.415 & $<0.001$ \\
Slope & 20.757 & 0.479 & 43.365 & $<0.001$ \\
\hline
\end{tabular}

(Fig. 2):

age $=-2.907+0.408 g+20.757\left(\frac{\mathrm{Bo}}{\mathrm{Ca}}\right)$

This model explained $86 \%$ of total variance $\left(R^{2}=0.86\right)$. The median of the absolute values of residuals (observed age minus predicted age) was 0.02 years, with a quartile deviation of 1.02 years, and a standard error of estimate of 0.96 years.

\section{Discussion}

In the last century the discovery of rays and the increasing number of auxological and forensic questions have encouraged the study of bone mineralisation as an age indicator.

$\mathrm{X}$-ray examination of the hand is one of the most widely used procedures for age estimation, and several methods have been developed to study the morphological parameters of hand and wrist for this purpose. One of the last is a method published in 2005 by Cameriere at al. which analysed the mineralisation of carpals. Results showed that the accuracy of chronological age estimations based on this new method is comparable with that of the FELS and TW3 methods, but it is easier to use than these techniques, and also shows a reduction in the betweensubject variability observed when skeletal maturity is evaluated by means of an atlas and other many qualitative predictors. The
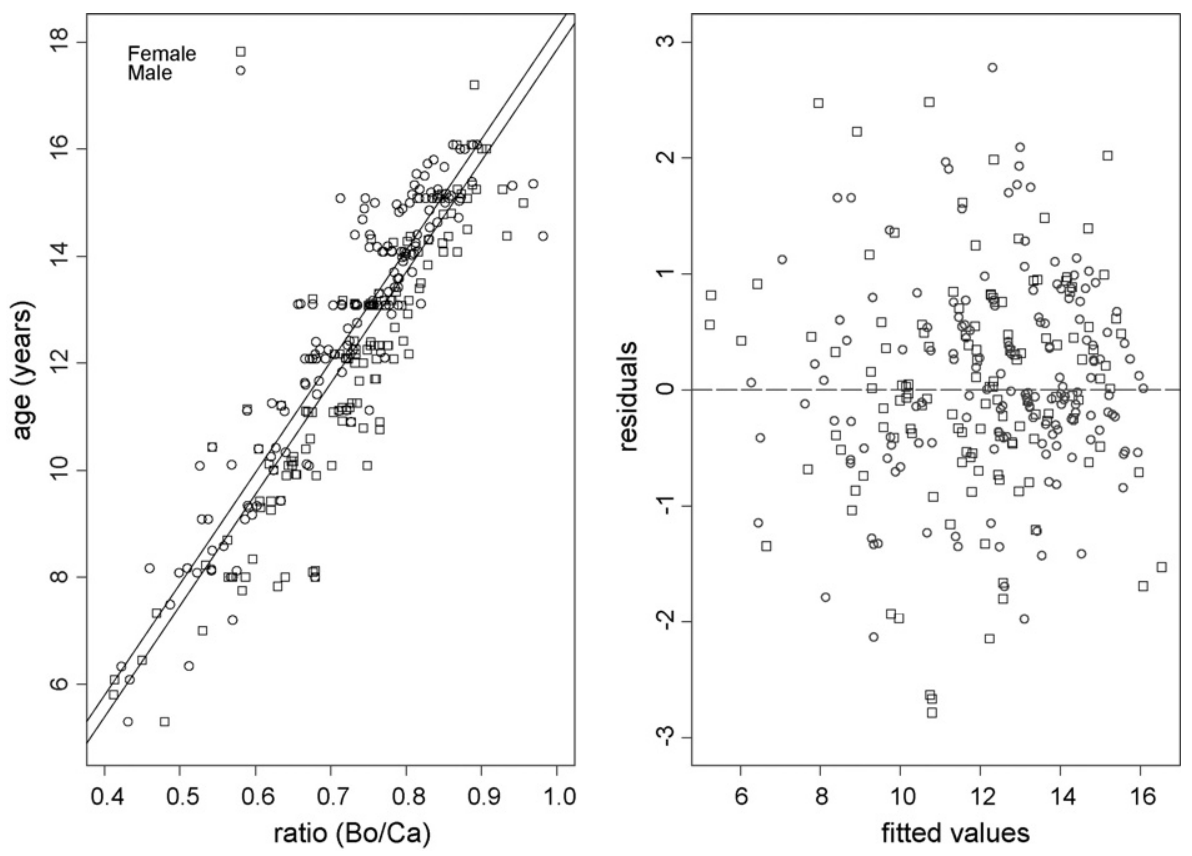

Fig. 2. Plots of data and regression lines (left panel) and residuals against fitted values (right panel) using a simple linear regression model (Eq. (2)). 
aim the present study was to test this method on a Slovenian sample. One of the most important problems in age estimation is the variation in different countries. Several methods, using both by teeth and wrist, are compared with different samples from various countries with interesting results. Nearly all studies highlight the fact that the original equation cannot be applied indifferently. The methods of age estimation that have been tested on the largest number of different nationalities are Demirjian method [14-17] and Greulich and Pyle method [1822], regarding teeth and wrist and hand, respectively.

Although the Greulich and Pyle atlas is the recommended method in all the international protocols and is considered as an acceptable standard, these studies found that the original formula was not applicable in different countries or between races. We therefore studied a new Slovenian sample, to obtain an original model and, then, we compared this equation with the equation obtained in Ref. [13].

The results did not reveal any statistically significant difference between the two countries and, therefore, suggested the possibility of applying one regression equation to both samples.

The yielded Eq. (2) will be also shown on the web site http:// agestimation.unimc.it. If researches on samples of different nationalities can be undertaken, results will call for new parameter estimations, and will be evaluated again in order to find a possible new equation with more general validity. If that is the case, the countries involved in the estimation procedure will be reported on the same web site to highlight the countries where the proposed formula can be used. The same project includes assessment of chronological age using teeth following the method described in Ref. [23]. Also in this case, the equation will be modified if the study of new samples will be needed, and the countries where the formula can be applied with are listed.

In cases when the sample is discordant with these of other countries, a single equation will be developed, and a new sample will be assessed to measure the discordance.

\section{References}

[1] J.M. Tanner, R.H. Whitehouse, Atlas of Children's Growth: Normal Variation and Growth Disorders, Academic Press, New York, 1982.

[2] P.A. Lee, S.S. Guo, H.E. Kulin, Age of puberty: data from the United States of America, APMIS 109 (2001) 81-88.

[3] D. Schulze, U. Rother, A. Fuhrmann, S. Richel, G. Faulmann, M. Heiland, Correlation of age and ossification of the medial clavicular epiphysis using computed tomography, Forensic Sci. Int. 158 (2006) 184-189.

[4] A. Schmeling, R. Schulz, W. Reisinger, M. Muhler, K.D. Wernecke, G. Geserick, Studies on the time frame for ossification of the medial clavicular epiphyseal cartilage in conventional radiography, Int. J. Legal Med. 118 (2004) 5-8.

[5] K.F. Kreitner, F.J. Schweden, T. Riepert, B. Nafe, M. Thelen, Bone age determination based on the study of the medial extremity of the clavicle, Eur. Radiol. 8 (1998) 1116-1122.

[6] S.I. Pyle, N.L. Hoerr, Radiographic Atlas of Skeletal Development of the Knee. A Standard of Reference, Charles C. Thomas, Spingfield, 1969.

[7] C.F.A. Moorees, E.A. Flamming, E.E. Hunt, Age variation of formation stages for ten permanent teeth, J. Dent. Res. 42 (1963) 1490.

[8] C.M. Nolla, The development of the permanent teeth, J. Dent. Child. 27 (1960) 254

[9] A. Demirjian, H. Goldstein, J.M. Tanner, A new system of dental age assessment, Hum. Biol. 5 (1973) 211.

[10] W.W. Greulich, S.I. Pyle, Radiographic Atlas of Skeletal Development of Hand and Wrist, 2nd ed., Stanford University Press, Stanford, California, 1959.

[11] J.M. Tanner, M.J.R. Healy, H. Goldstein, N. Cameron, Assessment of Skeletal Maturity and Prediction of Adult Height (TW3 Method), WD Saunders, London, UK, 2001.

[12] A.F. Roche, W. Cameron Chumlea, D. Thissen, Assessing the Skeletal Maturity of the Hand-wrist: Fels Method, Charles C. Thomas, Springfield, 1988.

[13] R. Cameriere, L. Ferrante, D. Mirtella, M. Cingolani, Carpals and epiphyses of radius and ulna as age indicators, Int. J. Legal Med. 120 (2005) 143-146.

[14] Z. Nyarady, H. Mornstad, L. Olasz, G. Szabo, Age estimation of children in south-western Hungary using the modified Demirjian method, Fogorv Sz. 98 (2005) 193-198.

[15] N. Chaillet, G. Willems, A. Demirjian, Dental maturity in Belgian children using Demirjian's method and polynomial functions: new standard curves for forensic and clinical use, J. Forensic Odontostomatol. 22 (2004) 1827.

[16] N. Chaillet, A. Demirjian, Dental maturity in South France: a comparison between Demirjian's method and polynomial functions, J. Forensic Sci. 49 (2004) 1059-1066.

[17] R.M. Eid, R. Simi, M.N. Friggi, M. Fisberg, Assessment of dental maturity of Brazilian children aged 6-14 years using Demirjian's method, Int. J. Paediatr. Dent. 12 (2002) 423-428.

[18] R.T. Loder, D.T. Estle, K. Morrison, D. Eggleston, D.N. Fish, M.L. Greenfield, K.E. Guire, Applicability of the Greulich and Pyle skeletal age standards to black and white children of today, Am. J. Dis. Child. 147 (1993) 1329-1333.

[19] R.R. Van Rijn, M.H. Lequin, S.G. Robben, W.C. Hop, C. Van Kuijk, Is the Greulich and Pyle atlas still valid for Dutch Caucasian children today? Pediatr. Radiol. 31 (2001) 748-752.

[20] J. Jimenez-Castellanos, A. Carmona, C.J. Catalina-Herrera, M. Vinuales, Skeletal maturation of wrist and hand ossification centers in normal Spanish boys and girls: a study using the Greulich-Pyle method, Acta Anat. 155 (1996) 206-211.

[21] S. Mora, M.I. Boechat, E. Pietka, H.K. Huang, V. Gilsanz, Skeletal age determinations in children of European and African descent: applicability of the Greulich and Pyle standards, Pediatr. Res. 50 (2001) 624-628.

[22] A. Koc, M. Karaoglanoglu, M. Erdogan, M. Kosecik, Y. Cesur, Assessment of bone ages: is the Greulich-Pyle method sufficient for Turkish boys? Pediatr. Int. 43 (2001) 662-665.

[23] R. Cameriere, L. Ferrante, M. Cingolani, Age estimation in children by measurement of open apices in teeth, Int. J. Legal Med. 120 (2006) 49-52. 